\title{
Muscle-related traits in cattle: The role of the myostatin gene in the South Devon breed
}

\author{
Pamela Wiener*, Judith Alexis SMith**, \\ Alyson Margaret Lewis***, John Arthur Woolliams, \\ John Lewis WiLliams \\ Roslin Institute, Roslin, Midlothian, EH25 9PS, UK
}

(Received 22 February 2001; accepted 19 October 2001)

\begin{abstract}
In this paper, we examined the effects of an 11-bp mutation within the GDF-8 gene, originally identified in Belgian Blue cattle, in the South Devon breed. The mutation was found at moderate frequency (0.37) in the South Devon population. We quantified the effects of this mutation on growth, body composition and calving traits in South Devon cattle. We found that the mutation significantly increased muscle score and calving difficulty and decreased fat depth. The mutation did not significantly affect weight at 200 and 400 days or muscle depth. Its effect on muscle score and fat depth was additive while its effect on calving difficulty was recessive. The mutation accounted for a significant proportion of the phenotypic variance in muscle score and calving difficulty. There was an economic benefit of the mutation for this data set, however, calculations were sensitive to changes in the parameter values. Additional data would be required to refine these calculations.
\end{abstract}

GDF -8 / myostatin / South Devon cattle / double muscling / muscle hypertrophy

\section{INTRODUCTION}

Identification of the myostatin gene $(G D F-8)$ has been one of the most important findings in cattle genetics of the last decade since it represents the first gene to have been identified with a large effect on an economically important trait. Variation in this gene, first discovered in mice [16] and then identified in cattle [10], has been shown to explain large phenotypic

\footnotetext{
* Correspondence and reprints

E-mail: Pam.Wiener@bbsrc.ac.uk

** Present address: Department of Veterinary Parasitology, University of Glasgow, Bearsdon Road, Glasgow, G61 1QH, UK.

*** Present address: Wellcome Centre for Molecular Parasitology, University of Glasgow, Dumbarton Road, Glasgow, G11 6NU, UK.
} 
differences between several breeds of cattle [11]. In particular, an allele with an 11-bp deletion, nt821(del11) [11] (hereafter called del11) has been associated with the phenotype described as "double muscling", an extreme form of muscle development characterized by a large increase in muscle mass (due primarily to muscle hyperplasia, an increase in the number of muscle cells). This particular mutation is found in a number of cattle breeds, including Belgian Blue, Parthenaise [11] and Asturiana de los Valles [7], and more recently, in the South Devon, where the frequency was found to be 0.39 [20]. The deletion is in the third exon of the gene and is a frameshift mutation resulting in the production of an inactive, truncated protein. Other mutations associated with increased muscling have also been found in this gene $[11,14,15]$.

Allele del11 in the South Devon is at intermediate frequency, and therefore, the genetic variation explained by $G D F-8$ is close to its peak. Furthermore, the allele frequency will respond rapidly to selection either up or down. This balance poses the critical question: what is the desired direction for selection in a breed with intermediate frequency? This question can only be answered with an assessment of the pleiotropic effects of the genotypes. Two additional but related issues are also relevant: (1) whether the $G D F-8$ locus has a significant impact on the overall genetic correlation between phenotypic traits, which has implications for the breed evaluation systems, and (2) whether non-additive variation is found for the $G D F-8$ locus, which could have implications for the optimal breed structure.

Therefore, the key information required is the way in which the del11 allele affects traits of economic importance. There is information available in the literature on the effects of double muscling on weight $[4,5,19]$, muscling [4, 21] and calving difficulty [5]. However, this information is limited in two ways. The first is that many results were derived prior to the identification of $G D F-8$ so that genotypes had to be inferred from phenotypes. As a result of this, del11/del11 individuals included in these studies were likely to be an unrepresentative sample of that genotype (i.e. more exaggerated phenotypically). The second is that these estimates were made mainly in experimental, crossbred animals (with no South Devon ancestry) and may not hold for the South Devon population.

This paper documents the effects of the del11 allele on many traits of economic importance in the South Devon and quantifies the multivariate impact of the del11 alleles on the traits recorded in a random sample of the breed. Additive genetic variation explained by $G D F-8$ is also estimated and compared to the parameters currently used for breed evaluation. Implications for selection strategies in the South Devon breed are assessed in light of the results of the analyses. 


\section{MATERIALS AND METHODS}

\subsection{Animals and traits}

A sample of 321 UK South Devon cattle born between 1984 and 1999 (with $60 \%$ born in 1998 or 1999) was identified. This sample comprised 244 females and 77 males, progeny of 80 sires from 20 farms. Ten of the sires had offspring on different farms and the average size of half-sib families was 4.1 (and ranged from 1 to 18 ). The bias in the sex ratio was due to the fact that many of the males in the population had already been slaughtered or sold on as calves by the time of recording. The animals included in the molecular analysis of Smith et al. [20] (107 total) were also included in the 321 in this study.

Animals in the sample were recorded for six phenotypic traits as part of the UK beef-recording scheme: 200-day weight, 400-day weight, muscle score, muscle depth, fat depth and calving difficulty. The weight data has been adjusted using standard Meat \& Livestock Commission (MLC) methods to correct for animals being weighed near to (but not at) the target age [6]. Muscle score (conformation) was assessed on a 15-point scale by professional inspectors of the MLC (Signet, Milton Keynes) with higher scores indicating greater muscle definition. Muscle and fat depth were measured by ultrasound scanning: muscle depth was measured at the 3rd lumbar vertebra and fat depth averaged from measurements at the 13th rib and 3rd lumbar vertebra. Calving difficulty was scored on a 5-point scale where 1 represents an easy, unassisted calving and 5 represents caesarian delivery. No data was recorded on stillborn animals and not all animals had measurements for all traits.

\subsection{Genotyping}

All animals were genotyped for the presence of del11 by visual inspection of PCR products resolved by agarose gel electrophoresis [20]. Allele and genotype frequencies were calculated directly from counts. The standard error of allele frequency was calculated with assumptions of binomial sampling [22].

\subsection{Statistical analysis}

The data on recorded traits were obtained from the MLC database and mixed linear models were fitted to analyze the effects of sex and genotype. The fixed effects were sex (male, female; $1 \mathrm{df})$, age of dam (1 df), birth year (11 df), genotype (del11/del11, del11/+, ++; $2 \mathrm{df})$, sex-genotype interaction ( $2 \mathrm{df})$ and farm (19 df).

In order to take into account that comparisons of paternal half-sibs should be weighted differently from unrelated animals, sires were included as a random effect. Since the sample size was relatively small, the proportion of the phenotypic variance attributable to sires was not estimated directly from the 
data in the principal analyses but was assumed known. The value assumed was $1 / 4 h^{2}$, where $h^{2}$ was the heritability for the trait calculated for the UK beef cattle population by Signet [6].

The models were fitted using the restricted maximum-likelihood (REML) option of Genstat [9]. Statistical significance for the fixed effects was determined by Wald tests and test statistics were compared to a $\chi^{2}$ distribution with the appropriate degrees of freedom. Wald tests rather than F-tests were used because the models were mixed (including both random and fixed effects) and are an approximation to a likelihood ratio test [9].

Sub-models were also fitted to explain the data in more detail. First, the effect of genotype was replaced in the model by a regression on allele number (i.e. number of del11 alleles in the genotype). The value of this regression coefficient, $\alpha_{i}$, is the effect of the allelic substitution for trait $i$. In order to test for dominance, an additional regression covariate was added with value 0 for homozygotes and 1 for heterozygotes. A significant result for this covariate was interpreted as evidence of a dominance effect. Further analyses estimated the sire variance directly from the data, rather than assuming it to be known, and the sire component was tested for significance using a likelihood ratio test comprising the log-likelihoods from analyses including and excluding the component from the model.

Other factors considered in the model were interactions of farm by genotype, farm by birth year and genotype by birth year, which were all treated as random effects.

\subsection{Genetic variances/covariances}

Allele frequencies and additive allelic effects were used to calculate the proportion of phenotypic variance explained by the $G D F-8$ gene and the genetic covariances for the four traits for which the effect of genotype were significant [8]. To do this, the effects of the allele substitution were standardized by the phenotypic variances obtained from the analyses, i.e. $\alpha_{i}^{\prime}=\frac{\alpha_{i}}{\sqrt{V_{P_{i}}}}$ (where $V_{P}$ included residual, genetic-due-to-sire and genetic-due-to- $G D F-8$ variance). The genetic variance explained by the myostatin locus for trait $i$ is given by $2 p q \alpha_{i}^{\prime 2}$ and the genetic covariance between traits $i$ and $j$ by $2 p q \frac{\alpha_{i}}{\sqrt{V_{P_{i}}}} \frac{\alpha_{j}}{\sqrt{V_{P_{i}}}}$ ( $p=$ the frequency of the del11 allele, $q=1-p$ ).

\section{RESULTS}

Allele frequencies in the sample were + : 0.63 and del11: 0.37 $\left(\mathrm{se}=1.09 \times 10^{-3}\right)$. Observed genotype frequencies (and Hardy-Weinberg 
expectations) were $+/+: 0.39(0.40),+/$ del11: 0.48 (0.46) and del11/del11: 0.13 (0.14), showing no detectable departures from Hardy-Weinberg equilibrium and consistent with the allele frequencies observed by Smith et al. [20] for a subset of this data. There was no significant difference in allele or genotype frequencies between males and females.

Results from REML analyses are shown in Table I. As there were no significant age of dam effects or sex-genotype interactions, they were dropped from the model for all subsequent analyses.

\subsection{Effects of genotype}

Genotype and allele number had significant effects on muscle score, fat depth, and calving difficulty but not on muscle depth or 200- and 400-day weights. There was good evidence for additivity of del11 for all traits except calving difficulty (there was no significant effect of heterozygosity when included in the allele number model). The effect of del11 on calving difficulty appeared recessive (heterozygosity was significant; $p<0.001$ ). The following values were obtained from the regression analysis for the average effect of a substitution of a single del11 allele: an increase in muscle score by 1.1 points, a reduction in fat depth by $4 \mathrm{~mm}$, and an increase in calving difficulty by 0.27 points.

\subsection{Other effects}

Sex had a significant effect on all traits such that males were heavier than females at both ages, had higher muscle scores, and had greater muscle and fat depth. There was also greater difficulty in calving males than females. Farm also had significant effects on all six traits. Sire had a significant effect on 200-day weight, muscle score and muscle depth and birth year had significant effects on 200-day weight, muscle score and fat depth.

\subsection{Genetic variances/covariances}

The proportions of phenotypic variance explained by the del11 allele and genetic covariances for the six traits are shown in Table II. For comparison, the overall heritabilities and genetic covariances calculated for British beef cattle [6] are also shown. The traits where genotype accounted for a largest proportion of the additive genetic variance were muscle score, where the estimate of the additive genetic variance due to $G D F-8$ genotype was actually greater than the overall heritability estimate, and calving difficulty, where $G D F$ 8 accounted for $90 \%$ of the additive genetic variation. $G D F-8$ accounted for $8 \%$ and $31 \%$ of the additive genetic variation for muscle and fat depth, respectively. The covariances between some trait pairs that are attributable to $G D F-8$ were 
Table I. Results from restricted maximum likelihood (REML) analysis of effects of GDF-8 genotype on six phenotypic traits. Means were adjusted under a model accounting for farm, sex, birth year, genotype or allele number and sire (also accounting for genotype-birth year, farm-birth year and farm-genotype interactions). The traits are the following: weight at 200 days (wt200), weight at 400 days (wt400), muscle score (msc), muscle depth (mdp), fat depth (fdp) and calving difficulty (cdiff). Approximate standard errors are also given. Significance of Wald statistic is given, based on $\chi^{2}$ distribution (see text). (Significance levels: ${ }^{* * * *}$ refers to significance at $P<0.0005$, $^{* *}$ at $P<0.005$ and ${ }^{*}$ at $\left.P<0.05\right)$.

\begin{tabular}{|c|c|c|c|c|c|c|c|}
\hline & \multicolumn{3}{|c|}{ Sex } & \multirow{2}{*}{$\begin{array}{l}\text { Allele number } \\
\text { Additive effect } \\
\quad \text { of del1 } 1\end{array}$} & \multicolumn{3}{|c|}{ Genotype } \\
\hline & $\begin{array}{l}\text { Male } \\
\text { mean }\end{array}$ & $\begin{array}{c}\text { Female } \\
\text { mean }\end{array}$ & $\begin{array}{c}\text { se } \\
\text { of difference }\end{array}$ & & $\begin{array}{c}\text { del11/del11 } \\
\text { mean } \pm \text { se }\end{array}$ & $\begin{array}{c}\text { del11/+ } \\
\text { mean } \pm \text { se }\end{array}$ & $\begin{array}{c}+/+ \\
\text { mean } \pm \text { se }\end{array}$ \\
\hline WT200 (kg) & 319.7 & 275.5 & $5.80^{* * *}$ & $3.27 \pm 7.06$ & $296.0 \pm 8.28$ & $300.3 \pm 4.75$ & $296.5 \pm 5.16$ \\
\hline WT400 (kg) & 604.6 & 418.2 & $12.87^{* * *}$ & $0.0668 \pm 15.96$ & $458.7 \pm 27.89$ & $448.9 \pm 12.19$ & $450.6 \pm 13.94$ \\
\hline MSC (1-15) & 9.31 & 6.08 & $0.20^{* * *}$ & $1.096 \pm 0.39^{* *}$ & $9.00 \pm 0.37^{* *}$ & $7.42 \pm 0.19$ & $6.67 \pm 0.21$ \\
\hline $\operatorname{MDP}(\mathrm{mm})$ & 75.04 & 57.82 & $0.96^{* * *}$ & $1.14 \pm 0.91$ & $66.85 \pm 2.75$ & $67.23 \pm 1.07$ & $65.21 \pm 1.14$ \\
\hline FDP (mm) & 31.02 & 25.66 & $1.55^{* *}$ & $-4.057 \pm 1.02^{* * *}$ & $23.80 \pm 2.29^{* * *}$ & $28.83 \pm 1.18$ & $32.40 \pm 1.26$ \\
\hline CDIFF (1-5) & 1.49 & 1.31 & $0.085^{*}$ & $0.27 \pm 0.098^{*}$ & $1.80 \pm 0.098^{* * *}$ & $1.24 \pm 0.051$ & $1.15 \pm 0.057$ \\
\hline
\end{tabular}


Table II. Genetic variances and covariances calculated for British beef cattle traits by Signet (above diagonal; Collins, [6]) and those calculated for effects of myostatin gene in South Devon breed (bold values below diagonal; this paper). Variances and covariances were standardized by phenotypic variances, thus genetic variances are heritabilities. The traits are the following: weight at 200 days (wt200), weight at 400 days (wt400), muscle score (msc), muscle depth (mdp), fat depth (fdp) and calving difficulty (cdiff).

\begin{tabular}{|c|c|c|c|c|c|c|}
\hline & WT200 & WT400 & MSC & MDP & FDP & CDIFF \\
\hline \multirow[t]{2}{*}{ WT200 } & 0.34 & +0.31 & +0.13 & +0.22 & +0.069 & +0.059 \\
\hline & 0.0053 & & & & & \\
\hline \multirow{2}{*}{ WT400 } & $+\mathbf{0 . 0 0 0 0 3 7}$ & 0.40 & +0.18 & +0.18 & +0.041 & +0.022 \\
\hline & & $2.64 \times 10^{-7}$ & & & & \\
\hline \multirow[t]{2}{*}{ MSC } & +0.040 & $+2.80 \times 10^{-4}$ & 0.26 & +0.17 & 0 & +0.035 \\
\hline & & & 0.296 & & & \\
\hline \multirow[t]{2}{*}{ MDP } & +0.010 & $+7.40 \times 10^{-5}$ & +0.078 & 0.26 & +0.049 & +0.035 \\
\hline & & & & 0.021 & & \\
\hline \multirow[t]{2}{*}{ FDP } & -0.022 & $-1.55 \times 10^{-4}$ & -0.164 & -0.043 & 0.29 & +0.089 \\
\hline & & & & & 0.091 & \\
\hline \multirow[t]{2}{*}{$\overline{\mathrm{CDIFF}}$} & +0.024 & $+1.69 \times 10^{-4}$ & +0.179 & +0.047 & -0.099 & 0.12 \\
\hline & & & & & & 0.108 \\
\hline
\end{tabular}

quite different from those accounted for by all additive genetic effects. For example, calving difficulty was strongly correlated with muscle score when $G D F-8$ genotype was considered but the current industry parameters assume this covariance to be very low. Nearly half of the genetic covariance between muscle depth and muscle score can be accounted for by myostatin genotype. Furthermore, covariances due to $G D F-8$ genotype between fat depth and both muscle score and calving difficulty were negative although the overall estimates of their genetic covariances were positive.

\section{DISCUSSION}

This study looked at a wide range of traits in a single (non-crossbred) population that has an 11-bp deletion (del11) segregating at the GDF-8 locus. The results of the study suggested that del11 had additive pleiotropic effects on muscle score and fat depth, with recessive behaviour observed for calving difficulty. The variation at $G D F-8$ explained a considerable portion of the genetic variation in muscling score and calving difficulty, and its pleiotropic effects 
explained substantial genetic covariances (with some covariances opposite to those generally assumed for UK beef breeds [6]).

There has been considerable information published on the impact of double muscling on performance and these publications may be roughly divided into two sets: (i) results of studies prior to 1990 where there was a recognition of a genetic influence on the occurrence of double muscling but there was no formal test of genotype (e.g., see refs. [12,13,17,19,21]; also reviewed by Arthur [3]); and (ii) results of studies where crossbred animals were directly genotyped $[4,5]$. While such studies act as benchmarks for the current study, the results of both sets need to be confirmed for a single population with a segregating del11 allele. In the earlier studies, phenotypic allocation to genotypes was based upon a visual appraisal of muscling score. Such an approach may lead to inaccurate estimates of allelic effects in populations with both $G D F-8$ alleles segregating. For example, in the top quartile for muscling score of the South Devon sample, the frequency of the del11 allele was 50\%. If it is assumed that the three genotypes had completely distinct phenotypic distribution with respect to muscling score, it would have been expected to find $78 \%$ del11 alleles in the top quartile (made up of all the homozygotes $14 \%$ of the population - and some heterozygotes - $11 \%$ of the population). With regard to more recent studies incorporating genotypic data, one needs to test whether the effects seen in experimental crossbreeds hold for a single population with segregation at the $G D F-8$ locus.

The differences between genotypes that we observed in live weight are overall consistent with both earlier groups of papers in that the difference in weights between $+/+$ and del11/ + was small at both 200 and 400 days. However, the del11/del11 homozygote weighed slightly more at 400 days, unlike the study of Casas et al. [5]. We found that the effect of dominance was not significant, but the study of Casas et al. [5] found statistically compelling evidence for dominance and this should be examined more closely in any further study of the South Devon.

The present study looked at predictors of carcass quality in all three genotypes, unlike the study of Casas et al. [4] where more detailed carcass traits were only measured in $+/+$ and del11/+ animals. Our study suggests that the del11 allele has additive effects on muscling score and fat depth. Casas et al. [4] found the heterozygote to have greater muscle area (longissimus rib eye) and retail yield, but decreased marbling, yield grade and carcass fat measures. The results for the comparison of the heterozygote and wild type for these traits are similar in our study and theirs. The inclusion of the del11/del11 homozygotes in examining the effects on muscle and fat traits provides important information because these traits are key predictors of carcass conformation and fat scores [6].

We found that the impact of the del11 allele on calving difficulty was recessive as has been reported previously by Casas et al. [5]. Reviewing 
our data on the South Devon, we found that that $45 \%$ (17/38) of del11/del11 calves required some form of assistance (42\% for females, $45 \%$ for males), compared to $11 \%(17 / 144)$ for heterozygotes ( $11 \%$ for females, $16 \%$ for males) and $7 \%(8 / 112)$ for $+/+$ homozygotes ( $6 \%$ for females, $14 \%$ for males). It is possible that the effect on calving difficulty was underestimated because no data was available on stillborn calves and any future studies might benefit from obtaining samples from stillbirths.

It has been suggested that calving difficulty may be a function of the dam's genotype as well as that of the calf [18]. Although we did not have genotypic information on the dams, predictions can be made using known genotype frequencies in the population. For the del11/+ calves, the expected frequencies of maternal genotypes are $p / 2: 0.5:(1-p) / 2$ for del11/del11, del11/ + , and $+/+$, respectively (where $p$ is the frequency of the del11 allele). For the $+/+$ calves, the frequencies of maternal genotypes are $0: p: 1-p$. For this population, $p=0.37$ so that nearly $20 \%$ of the del11/+ calves will have been produced by del11/del11 dams compared to none for the $+/+$ calves. If there were a substantial effect of the del11/del11 maternal genotype, one might have expected to see it in the comparison of calving difficulty between del11/+ and $+/+$ individuals. However, these two groups were very similar, supporting the view that the calf's myostatin genotype is more influential than that of its dam in determining the difficulty of calving.

The objective of this study was to review the impact of the del11 allele for breeds in which it is segregating. The MLC, which provides technical services for UK beef cattle breeders, use two indices: (i) Beef Value, intended to target improvements in carcass weight and conformation while maximizing farm profitability [1], and (ii) Calving Value, intended to target improvements in gestation length and calving ease. The genetic parameters and the economic values used for these indices are described in detail by Amer and Simm [2]. Using the above results for the different genotypes, we predicted the impact on Beef and Calving Values.

Considering both Beef Value and Calving Value, there is a net gain of the del11 allele in a segregating beef population. The impact on Beef Value of a del11 allelic substitution was predicted to be $0.04,0.54$ and 0.59 genetic standard deviations for carcass weight, carcass conformation, and carcass fat, respectively. This represents an increase in Beef Value due to the allelic substitution (del11 for + ) of 9.57 euros (roughly $24 \%$ of the profit margin) per calf. For Calving Value, the same allelic substitution is predicted to change gestation length and calving ease by 0.38 and 0.76 genetic standard deviations, representing a loss in Calving Value of 5.52 euros (roughly 14\% of the profit margin) per calf due to the allelic substitution. Therefore, there is a net gain of the del11 allele (of 4.05 euros per calf) at its current frequency, and in fact, at all frequencies. However this initial conclusion needs to be considered with some 
caution since (i) not all traits are additive, (ii) the Beef and Calving Values for the del11 substitution are not known without error, and (iii) these calculations were found to be highly sensitive to changes in the parameter values. Regarding the last point, a relatively small change in the effect of genotype on weight traits drastically reduced the Beef Value, and the resulting net value was negative. This sensitivity resulted in part from the small sample size of this experiment and emphasizes the need for larger data sets in order to accurately measure the economic value of this gene. It should also be noted that the phenotypic effects and hence the economic impact are likely to vary depending on the genetic background, which differs between breeds. Furthermore, because del11 affects calving difficulty, there are potentially issues related to animal welfare and public attitudes that should also be considered, independent of the economic value of the allele in the current market.

In conclusion, this study has shown that the segregating alleles at the myostatin gene have significant effects on carcass characteristics and calving ease in the South Devon breed. The del11 has both positive and negative effects on traits of economic and welfare importance, therefore some assessment needs to be made by breeders as to the best way forward.

\section{ACKNOWLEDGEMENTS}

We gratefully acknowledge assistance in obtaining samples and phenotypic information from Duncan Pullar and Barry Weigleb (Meat \& Livestock Commission), Rob Shields (Signet) and the South Devon Breed Society (especially Lesley Lewin). This project was funded by the Meat \& Livestock Commission.

\section{REFERENCES}

[1] Amer P.R., Emmans G.C., Simm G., Economic values for carcass traits in UK commercial beef cattle, Livest. Prod. Sci. 51 (1997) 267-281.

[2] Amer P.R., Simm G., An index to assist multiple trait selection of terminal beef sires in the UK, Proceedings of the New Zealand Society of Animal Production 57 (1997) $15-18$.

[3] Arthur P.F., Double muscling in cattle: a review, Aust. J. Agric. Res. 46 (1995) 1493-1515.

[4] Casas E., Keele J.W., Shackleford S.D., Koohmaraie M., Sonstegard T.S., Smith T.P.L., Kappes S.M., Stone R.T., Association of the muscle hypertrophy locus with carcass traits in beef cattle, J. Anim. Sci. 76 (1998) 468-473.

[5] Casas E., Keele J.W., Fahrenkrug S.C., Smith T.P.L., Cundiff L.V., Stone R.T., Quantitative analysis of birth, weaning, and yearling weights and calving difficulty in Piedmontese crossbreds segregating an inactive myostatin allele, J. Anim. Sci. 77 (1999) 1686-1692. 
[6] Collins J.M.E., Genetic evaluation and selection of purebred beef cattle in the UK, Base Paper, Signet, Milton Keynes, UK, 1999.

[7] Dunner S., Charlier C., Farnir F., Brouwers B., Canon J., Georges M., Towards interbreed IBD fine mapping of the $m h$ locus: double-muscling in the Asturiana de los Valles breed involves the same locus as in the Belgian Blue cattle breed, Mamm. Genome 8 (1997) 430-435.

[8] Falconer D.S., Mackay T.F.C., Introduction to Quantitative Genetics, Fourth Edition, Addison Wesley Longman Limited, Harlow, 1996.

[9] Genstat 5 Committee of the Statistics Department, Rothamsted Experimental Station, Genstat 5 Release 4.1 Reference Manual, Clarendon Press, Oxford, 1998.

[10] Grobet L., Martin L.J.R., Poncelet D., Pirottin D., Brouwers B., Riquet J., Schoeberlein A., Dunner S., Ménissier F., Massabanda J., Fries R., Hanset R., Georges M., A deletion in the bovine myostatin gene causes the double-muscled phenotype in cattle, Nature Genet. 17 (1997) 71-74.

[11] Grobet L., Poncelet D., Royo J.L., Brouwers B., Pirottin D., Michaux C., Ménissier F., Zanotti M., Dunner S., Georges M., Molecular definition of an allelic series of mutations disrupting the myostatin function and causing doublemuscling in cattle, Mamm. Genome 9 (1998) 210-213.

[12] Hanset R., Muscular hypertrophy as a racial characteristic: The case of the Belgian Blue breed, in: King J.W.B., Ménissier F. (Eds.), Muscle Hypertrophy of Genetic Origin and its use to Improve Beef Production, Martinus Nijhoff Publishers, London, 1982, pp. 437-449.

[13] Hanset R., Michaux C., On the genetic determinism of muscular hypertrophy in the Belgian White and Blue cattle breed. I. Experimental data, Genet. Sel. Evol. 17 (1985) 359-368.

[14] Kambadur R., Sharma M., Smith T.P.L., Bass J.J., Mutations in myostatin (GDF8 ) in double-muscled Belgian Blue and Piedmontese cattle, Genome Res. 7 (1997) 910-915.

[15] McPherron A.C., Lee S.-J., Double-muscling in cattle due to mutations in the myostatin gene, Proceedings of the National Academy of Science USA 94 (1997) 12457-12461.

[16] McPherron A.C., Lawler A.M., Lee S.-J., Regulation of skeletal muscle mass in mice by a new TGF- $\beta$ superfamily member, Nature 387 (1997) 83-90.

[17] Ménissier F., General survey of the effect of double muscling on cattle performance, in: King J.W.B., Ménissier F. (Eds.), Muscle Hypertrophy of Genetic Origin and its use to Improve Beef Production, Martinus Nijhoff Publishers, London, 1982, pp. 23-53.

[18] Murray R.D., Cartwright T.A., Downham D.Y., Murray M.A., Some maternal factors associated with dystocia in Belgian Blue cattle, Anim. Sci. 69 (1999) $105-113$.

[19] Nott C.F.G., Rollins W.C., Effects of the $m$ gene for muscular hypertrophy on birth weight and growth to one year of age in beef cattle, Growth 43 (1979) 221-234.

[20] Smith J.A., Lewis A.M., Wiener P., Williams J.L., Genetic variation in the bovine myostatin gene in UK beef cattle: Allele frequencies and haplotype analysis in the South Devon, Anim. Genet. 31 (2000) 306-309. 
[21] Thiessen R.B., Rollins W.C., A comparison of normal and heterozygous animals for double muscling in British breeds of cattle, in: King J.W.B., Ménissier F. (Eds.), Muscle Hypertrophy of Genetic Origin and its use to Improve Beef Production, Martinus Nijhoff Publishers, London, 1982, pp. 54-69.

[22] Weir B.S., Genetic Analysis II. Sinauer Associates, Inc., Sunderland, Massachusetts, 1996.

To access this journal online: www.edpsciences.org 